\title{
General approaches in ensemble quantum computing
}

\author{
V VIMALAN and N CHANDRAKUMAR* \\ Department of Chemistry, Indian Institute of Technology Madras, Chennai 600036 \\ e-mail: nckumar@iitm.ac.in
}

\begin{abstract}
We have developed methodology for NMR quantum computing focusing on enhancing the efficiency of initialization, of logic gate implementation and of readout. Our general strategy involves the application of rotating frame pulse sequences to prepare pseudopure states and to perform logic operations. We demonstrate experimentally our methodology for both homonuclear and heteronuclear spin ensembles. On model two-spin systems, the initialization time of one of our sequences is three-fourths (in the heteronuclear case) or one-fourth (in the homonuclear case), of the typical pulsed free precession sequences, attaining the same initialization efficiency. We have implemented the logical SWAP operation in homonuclear AMX spin systems using selective isotropic mixing, reducing the duration taken to a third compared to the standard re-focused INEPT-type sequence. We introduce the 1D version for readout of the rotating frame SWAP operation, in an attempt to reduce readout time. We further demonstrate the Hadamard mode of ID SWAP, which offers $2^{\mathrm{N}}$-fold reduction in experiment time for a system with $\mathrm{N}$-working bits, attaining the same sensitivity as the standard 1D version.
\end{abstract}

Keywords. JCP; PRAWN; pseudopure state; logic gate; isotropic mixing; Hadamard mode; quantum state tomography.

\section{Introduction}

NMR Quantum computation comprises three steps, viz. initialization of the spin ensemble, computation and readout of results. We have developed and implemented methodology in an attempt to enhance the efficiency of all three steps. Efficiency in this context refers both to the step duration and, perhaps even more significantly, to sensitivity or robustness to noise, governed in part by relaxation losses. Rotating frame sequences ${ }^{1}$ are known in general to differ from their Pulsed Free Precession (PFP) counterparts in coherence transfer (CT) and relaxation characteristics. Favourable CT rates, CT amplitudes and relaxation rates may result in enhanced efficiency with rotating frame sequences. Our general approach therefore involves the design and implementation of rotating frame sequences to initialize the spin ensemble and to implement logic operations.

The generation of pseudopure states is desirable in initializing a spin ensemble for quantum computing. ${ }^{2-4}$ When we deal with heteronuclear spin systems it is convenient to first prepare the symmetric state from the thermal state; the pseudopure state may then be prepared from the symmetric state. Our approach explores the implementation of $J$-cross pola-

\footnotetext{
*For correspondence
}

rization $(J \mathrm{CP})$ to create symmetric density matrices in a two-spin heteronuclear spin system; by further application of JCP, we seek to prepare the pseudopure state. In the case of homonuclear spin systems, where the thermal state is symmetric, we explore isotropic mixing to prepare the pseudopure state directly from the thermal state. We show that in both systems, the 'initialization' time is reduced for a given amplitude of the resulting pseudopure state. The pseudopure state thus generated may be visualized by quantum state tomography ${ }^{5,6}$ and may further be employed to run a quantum algorithm, e.g. Grover's algorithm. ${ }^{7,8}$

The logic operation SWAP ${ }^{9,10}$ performed on coupled spins (I-S) interchanges the $\mathrm{I}$ and $\mathrm{S}$ spins in any given product operator. This permits execution of a conditional NOT (C-NOT) or exclusive OR (XOR) logic gate between spins that are not coupled to each other. While the original implementations of SWAP were performed with a series of bilinear rotations, it seems natural to seek to implement SWAP by isotropic mixing, the relevant mixing Hamiltonian being equivalent to a permutation operator. ${ }^{11,12}$ Indeed, this has been shown to lead to reduction of the 'gating time' to one-third, working on a twospin-1/2 system. ${ }^{13}$ We have implemented the logical SWAP operation on the homonuclear AMX spin system using selective isotropic mixing. We show 
that the gating time is reduced to one-third in this case as well, to $(1 / 2 J)$ when compared with the standard sequence which has the gating time $(3 / 2 J), J$ being the coupling constant between the computation spins. The resulting significant gain in robustness to noise is to be underscored, especially for homonuclear ${ }^{1} \mathrm{H}$ systems, the gate duration being reduced by as much as $140 \mathrm{~ms}$ (for $J \sim 7 \mathrm{~Hz}$ ), resulting typically in at least $20 \%$ sensitivity enhancement. The implications for a cascade of steps could be very significant indeed.

The SWAP operation may be readout experimentally by performing a $2 \mathrm{D}$ experiment, which places obvious demands on experiment time. While ultrafast 2D implementations ${ }^{14}$ could be adapted for quantum computing, here we have developed a 1D analog ${ }^{15}$ of the 2D SWAP readout, resulting in considerable reduction in readout time. In the 1D mode we employ transition selective pulses to excite the multiplet components of the observer spin (ancilla bit) individually and perform selective isotropic mixing on the operational or computation spins. This 1D implementation of SWAP readout typically reduces the readout time by two orders of magnitude in comparison with the conventional $2 \mathrm{D}$ mode. In an attempt to further enhance the sensitivity or reduce the time of readout we have developed another set of experiments ${ }^{15}$ with Hadamard ${ }^{16}$ encoding and decoding. The excitation of the multiplet components of the observer spin is now phase encoded in keeping with the rows of the Hadamard matrix. The resulting spectra are then decoded in accordance with the columns of the Hadamard matrix. For fixed experiment time this mode offers sensitivity improvement by a factor of $\sqrt{M}$, where $M$ is the number of multiplet components involved, which is $2^{\mathrm{N}}$ for spin-1/2 systems, $N$ being the number of working bits (qubits or computational spins)

\section{Theory and experiments}

In the following, we summarize the theoretical considerations and pulse sequence design for each of a number of the steps involved in quantum computing. All experiments were performed on a Bruker Avance $400 \mathrm{MHz}$ NMR spectrometer system.

\subsection{Preparation of pseudopure states}

2.1a Heteronuclear two-spin-1/2 spin systems: We have chosen a heteronuclear two spin-1/2 system to implement our proposed rotating frame sequence. In this heteronuclear AX spin system the thermal state is $\left[4 I_{z}+S_{z}\right]$, where $I$ represents the ${ }^{1} \mathrm{H}$ spin and $S$ represents ${ }^{13} \mathrm{C}$. We first prepare a symmetric state $(5 / 2)\left[I_{z}+S_{z}\right]$ from the above unsymmetrical thermal state by employing $J$-cross polarization. ${ }^{15,17}$ With this symmetric state as the starting point, we prepare the pseudopure state, $a\left[I_{z}+S_{z}+2 I_{z} S_{z}\right]$, by using another $J C P$ module. The pulse sequence developed by us is shown in figure 1 .

The functioning of our pulse sequence may be readily visualized by a density matrix calculation. Segment $\mathbf{A}$ of the pulse sequence gives rise to:

$$
4 I_{z}+S_{z} \equiv \frac{5}{2}\left(I_{z}+S_{z}\right)+\frac{3}{2}\left(I_{z}-S_{z}\right) \stackrel{90_{x}^{\circ}(I, S)}{\longrightarrow}
$$

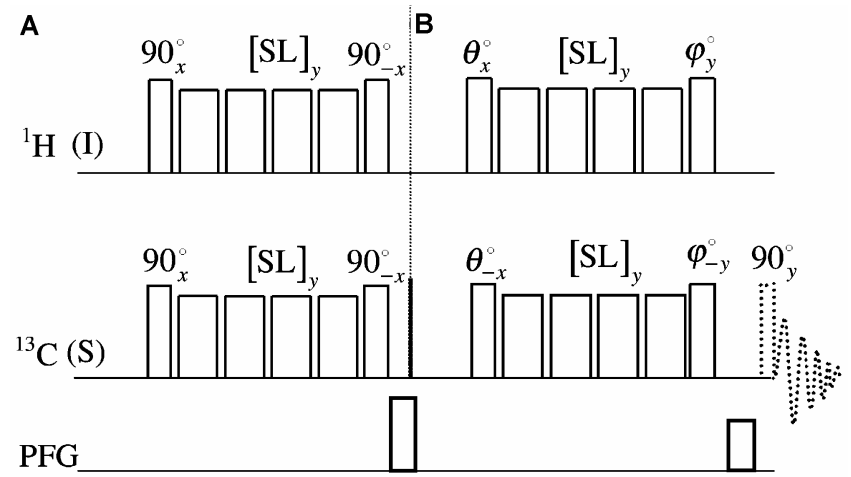

Figure 1. Pulse sequence to prepare pseudopure state from thermal state. The sequence is divided into two segments $\mathbf{A}$ and $\mathbf{B}$. Segment $\mathbf{A}$ of the sequence converts the unsymmetric thermal state of the $\mathrm{CH}$ system $\left[4 I_{z}+S_{z}\right]$ into a symmetric state $(5 / 2)\left[I_{z}+S_{z}\right]$. Spin lock is performed by using the PRAWN mixing sequence, which is highly selective and has low rf power deposition. The duration of the pulses in the spin lock train is $50 \mu \mathrm{s}$ for the ${ }^{13} \mathrm{C}$ and ${ }^{1} \mathrm{H}$ channels, the pulse interval being $203.5 \mu \mathrm{s}$. The mixing time is $(1 / 2 J) \mathrm{s}$, which in this case is $2.28 \mathrm{~ms}$. For the $90^{\circ}$ hard pulse $10 \mu$ s duration is employed for both ${ }^{1} \mathrm{H}$ and ${ }^{13} \mathrm{C}$ channels. $z$-gradient of $1 \mathrm{~ms}$ duration and amplitude $8.67 \mathrm{G} / \mathrm{cm}$ was used. Segment B of the sequence converts the symmetric state into a pseudopure state. The JCP sequence is the same as above. The mixing time here is $\left(1 / 4 J_{A X}\right)$ or $\left(1 / 2 \cdot 16 J_{A X}\right)$, depending upon the flip angles $\theta$ and $\varphi$. $z$-gradient of $1 \mathrm{~ms}$ duration with amplitude $22.36 \mathrm{G} / \mathrm{cm}$ was employed. In homonuclear spin systems the thermal state is symmetric and the first segment of the sequence, $\mathbf{A}$, is not required. We incorporate isotropic mixing instead of $J \mathrm{CP}$ with the mixing time $(1 / 8 J)$ or $(1 / 4 J)$ for two different sets of $\theta$ and $\varphi$. Generation of the pseudopure state may be demonstrated by applying the last (dotted) pulse and acquiring data. The readout is performed by a hard $90^{\circ}$ pulse on ${ }^{13} \mathrm{C} /{ }^{1} \mathrm{H}$ channel, whereas in the case of homonuclear spin systems it is a spin selective $90^{\circ}$ pulse. 


$$
\begin{aligned}
& -\frac{5}{2}\left(I_{y}+S_{y}\right)-\frac{3}{2}\left(I_{y}-S_{y}\right) \stackrel{(S L)_{y},(2 J)^{-1}}{\longrightarrow} \\
& -\frac{5}{2}\left(I_{y}+S_{y}\right)+3\left(I_{x} S_{z}-I_{z} S_{x}\right) \stackrel{90_{-x}^{\circ}(I, S)}{\longrightarrow} \\
& \frac{5}{2}\left(I_{z}+S_{z}\right)+3\left(I_{x} S_{y}-I_{y} S_{x}\right) \stackrel{(g r a d)_{z}}{\longrightarrow} \frac{5}{2}\left(I_{z}+S_{z}\right)
\end{aligned}
$$

Segment $\mathbf{B}$ of the sequence leads to:

$$
\begin{aligned}
& \frac{5}{2}\left(I_{z}+S_{z}\right) \stackrel{\theta_{x}(I), \theta_{-x}(S)}{\longrightarrow} \frac{5}{2}\left[\begin{array}{l}
\left(I_{z}+S_{z}\right) \cos \theta- \\
\left(I_{y}-S_{y}\right) \sin \theta
\end{array}\right] \\
& \stackrel{(S L)_{y}(I, S), t}{\longrightarrow} \frac{5}{2}\left[\begin{array}{l}
\left(I_{z}+S_{z}\right) \cos \theta \cos \left(\frac{\pi J t}{2}\right)- \\
2\left(I_{y} S_{x}+I_{x} S_{y}\right) \cos \theta \sin \left(\frac{\pi J t}{2}\right) \\
-\left(I_{y}-S_{y}\right) \sin \theta \cos \pi J t+ \\
2\left(I_{x} S_{z}-I_{z} S_{x}\right) \sin \theta \sin \pi J t
\end{array}\right] \\
& \stackrel{\varphi_{y}(I), \varphi_{-y}(S)}{\longrightarrow} \frac{5}{2}\left[\begin{array}{l}
\left(I_{z}+S_{z}\right) \cos \theta \cos \varphi \cos \left(\frac{\pi J t}{2}\right)+ \\
\left(I_{x}-S_{x}\right) \cos \theta \sin \varphi \cos \left(\frac{\pi J t}{2}\right) \\
-2\left(I_{y} S_{x}+I_{x} S_{y}\right) \cos \theta \cos \varphi \sin \left(\frac{\pi J t}{2}\right)- \\
\left(I_{y}-S_{y}\right) \sin \theta \cos \pi J t \\
-2\left(I_{y} S_{z}-I_{z} S_{y}\right) \cos \theta \sin \varphi \sin \left(\frac{\pi J t}{2}\right) \\
+2\left(\left(I_{x} S_{z}-I_{z} S_{x}\right) \cos 2 \varphi-\left(I_{x} S_{x}+I_{z} S_{z}\right)\right. \\
\sin 2 \varphi) \sin \theta \sin \pi J t
\end{array}\right]
\end{aligned}
$$$$
\stackrel{(\mathrm{grad})_{z}}{\longrightarrow} \frac{5}{2}\left[\begin{array}{l}
\left(I_{z}+S_{z}\right) \cos \theta \cos \varphi \cos \left(\frac{\pi J t}{2}\right)- \\
2 I_{z} S_{z} \sin \theta \sin 2 \varphi \sin \pi J t
\end{array}\right]
$$

The final density matrix at the end of the pulse sequence is given in (2a). This state corresponds to a pseudopure state provided the following condition is satisfied:

$$
\tan \theta \sin \varphi \sin \left(\frac{\pi J t}{2}\right)=\frac{1}{4}
$$

By variation of the parameters of segment $\mathbf{B}$ of the sequence of figure 1, viz. the flip angles $\theta, \varphi$ and the spin lock duration $t$ of (2a), while ensuring that (2b) is satisfied, we obtain pseudopure states of different amplitudes. While a fairly large set of choices of $(\theta$, $\varphi, t)$ lead to similar resulting amplitudes, we chose three settings for segment $\mathbf{B}$ of the sequence of fig- ure 1 , for which the spin lock duration is relatively short and the resulting pseudopure state amplitude is comparable with the laboratory frame sequences. ${ }^{3,4}$

The amplitudes of the pseudopure state created by these three choices for segment $\mathbf{B}$ of our rotating frame sequences, labelled SL-1a, SL-2a and SL-3a are shown in table 1 for different values of $\theta, \varphi$ and $t$. Table 1 also exhibits relevant parameters of the standard PFP sequences. ${ }^{3,4}$ The effect of relaxation on amplitudes has been ignored for the purposes of this comparison.

The sequence SL-la results in essentially the same amplitude as does the corresponding laboratory frame sequence PFP-la, but takes half the time to transform the symmetric state to the pseudopure state. This implies that the total initialization time with the rotating frame sequence of figure 1 , incorporating SL-la for segment $\mathbf{B}$ is three-fourths that of the corresponding laboratory frame approach, both approaches requiring a time $(2 J)^{-1}$ to first transform the thermal state to a symmetric state with equal efficiency.

Our rotating frame sequence SL-2a for segment B, on the other hand, requires $66.67 \%$ of the mixing time of the corresponding laboratory frame sequence, PFP-2a, while leading to $86.6 \%$ of its resulting amplitude. Once again, both rotating and laboratory frame versions require $(2 J)^{-1}$ to first transform the thermal state to a symmetric state with equal efficiency.

The implication of a pseudopure state amplitude of 0.5 is that the two-spin-1/2 diagonal density matrix has elements proportional to $2.5(0.25,0.25,0.25$, $-0.75)$. This is to be contrasted with the thermal state diagonal density matrix for the ${ }^{13} \mathrm{CH}$ system where the elements are proportional to $(2.5,1.5,-1.5,-2.5)$ and the corresponding symmetric state diagonal density matrix where elements are proportional to $(2 \cdot 5,0,0,-2 \cdot 5)$, the proportionality constants being identical for the three states in question.

Table 1. Two different rotating frame sequences to generate pseudopure states from symmetric states and comparison with corresponding laboratory frame sequences for heteronuclear two-spin-1/2 systems.

\begin{tabular}{lcccc}
\hline $\begin{array}{l}\text { Pulse } \\
\text { sequence }\end{array}$ & $\theta$ & $\varphi$ & $\begin{array}{c}\text { Mixing } \\
\text { time }(t)\end{array}$ & Amplitude \\
\hline SL-1a & $45^{\circ}$ & $40 \cdot 79^{\circ}$ & $1 /(4 J)$ & 0.4946 \\
SL-2a & $45^{\circ}$ & $30^{\circ}$ & $1 /(3 J)$ & 0.5303 \\
SL-3a & $40^{\circ}$ & $33^{\circ}$ & $1 /(2 \cdot 73 J)$ & 0.5362 \\
PFP-1a & Sandwich & $45^{\circ}$ & $1 /(2 J)$ & $0 \cdot 5$ \\
PFP-2a & $45^{\circ}$ & $30^{\circ}$ & $1 /(2 J)$ & $0 \cdot 6123$ \\
\hline
\end{tabular}


The implementation of a quantum algorithm generally starts with the initialization of the spin ensemble. Starting from thermal equilibrium and incorporating our methodology for initialization by generating pseudopure states, we have implemented Grover's search algorithm ${ }^{7,8}$ on a heteronuclear two spin-1/2 system. The standard pulse sequence ${ }^{8}$ for the implementation of Grover's algorithm is shown below, starting with the pseudopure state PPS:

$$
\begin{aligned}
& \text { PPS }-90_{y}-180_{-x}-\frac{1}{2 J}-90_{y}- \\
& 90_{\phi_{1}}^{I}, 90_{\phi_{2}}^{S}-\frac{1}{2 J}-90_{y}-90_{x}-\text { Grad }-90_{y}^{I / S}-\text { Acq. }
\end{aligned}
$$

The sequence involves an 'inversion about average' operation, implemented by the pulse sandwich that is flanked by delays of $(2 J)^{-1}$. Four scans are performed with different values of $\phi_{1}$ and $\phi_{2}$. The four different values of $\phi_{1}$ and $\phi_{2}$ for performing the inversion operation are shown in table 2 . The readout is performed on ${ }^{13} \mathrm{C}$ and ${ }^{1} \mathrm{H}$ spins. The result from execution of Grover's algorithm is interpreted in terms of the relative phases of the multiplet components of the spins detected on both channels. ${ }^{8}$

\subsection{Pseudopure state preparation for homonuclear two-spin-1/2 systems}

2.2a Basic pulse sequences: The thermal equilibrium density matrix for homonuclear spin systems is symmetric. The generation of a pseudopure state does not therefore require any prior symmetric state preparation unlike the situation with heteronuclear spin systems. The pulse sequence shown in figure 1 may therefore be used without segment $\mathbf{A}$ of the sequence, employing isotropic mixing instead of $J$ cross polarization (planar mixing) in segment $\mathbf{B}$. The

Table 2. The phases $\phi_{1}$ and $\phi_{2}$ shown in the pulse sequence for the implementation of Grover's algorithm (pulse sequence as in text).

\begin{tabular}{lrc}
\hline & \multicolumn{2}{c}{ Phase } \\
\cline { 2 - 3 } State & $\phi_{1}$ & $\phi_{2}$ \\
\hline$|00\rangle$ & $x$ & $x$ \\
$|01\rangle$ & $-x$ & $x$ \\
$|10\rangle$ & $x$ & $-x$ \\
$11\rangle$ & $-x$ & $-x$ \\
\hline
\end{tabular}

spin evolution and condition for generation of pseudopure state are shown in (3a) and (3b).

$$
\begin{aligned}
& \frac{5}{2}\left(I_{1 z}+I_{2 z}\right) \stackrel{\theta_{x}\left(I_{1}\right), \theta_{-x}\left(I_{2}\right)}{\longrightarrow} \\
& \frac{5}{2}\left[\left(I_{1 z}+I_{2 z}\right) \cos \theta-\left(I_{1 y}-I_{2 y}\right) \sin \theta\right] \\
& \stackrel{(S L)_{y}\left(I_{1}, I_{2}\right), t}{\longrightarrow} \frac{5}{2}\left[\begin{array}{l}
\left(I_{1 z}+I_{2 z}\right) \cos \theta- \\
\left(I_{1 y}-I_{2 y}\right) \sin \theta \cos 2 \pi J t+ \\
2\left(I_{1 x} I_{2 z}-I_{1 z} I_{2 x}\right) \sin 2 \pi J t
\end{array}\right]
\end{aligned}
$$$$
\stackrel{\varphi_{y}\left(I_{1}\right), \varphi_{-y}\left(I_{2}\right)}{\longrightarrow} \frac{5}{2}\left[\begin{array}{l}
\left(I_{1 z}+I_{2 z}\right) \cos \theta \cos \varphi+ \\
\left(I_{1 x}-I_{2 x}\right) \cos \theta \sin \varphi- \\
\left(I_{1 y}-I_{2 y}\right) \sin \theta \cos 2 \pi J t \\
+2\left(\begin{array}{l}
I_{1 x} I_{2 z} \cos 2 \varphi- \\
I_{1 x} I_{2 x} \sin 2 \varphi \\
-I_{1 z} I_{2 z} \sin 2 \varphi \\
-I_{1 z} I_{2 x} \cos 2 \varphi
\end{array}\right) \sin \theta \sin 2 \pi J t
\end{array}\right]
$$$$
\stackrel{(\text { grad })_{z}}{\longrightarrow} \frac{5}{2}\left[\begin{array}{l}
\left(I_{1 z}+I_{2 z}\right) \cos \theta \cos \varphi- \\
2 I_{1 z} I_{2 z} \sin \theta \sin 2 \varphi \sin 2 \pi J t
\end{array}\right]
$$

$$
\tan \theta \sin \varphi \sin 2 \pi J t=\frac{1}{2}
$$

By variation of the parameters of segment $\mathbf{B}$ of the sequence of figure 1, viz. the flip angles $\theta, \varphi$ and the spin lock duration $t$ of (3a), while ensuring that (3b) is satisfied, we obtain pseudopure states of different amplitudes. While a fairly large set of choices of $(\theta$, $\varphi, t)$ lead to similar resulting amplitudes, we chose three settings as in table 3 for segment $\mathbf{B}$ of the se-

Table 3. Two different rotating frame sequences to generate pseudopure states and comparison with corresponding laboratory frame sequences for homonuclear two-spin-1/2 systems.

\begin{tabular}{lcccc}
\hline $\begin{array}{l}\text { Pulse } \\
\text { sequence }\end{array}$ & $\theta$ & $\varphi$ & $\begin{array}{l}\text { Mixing } \\
\text { time }(t)\end{array}$ & Amplitude \\
\hline SL-1b & $45^{\circ}$ & $45^{\circ}$ & $1 /(8 J)$ & $0 \cdot 5$ \\
SL-2b & $50^{\circ}$ & $36 \cdot 4^{\circ}$ & $1 /(8 J)$ & $0 \cdot 5174$ \\
SL-3b & $42^{\circ}$ & $34^{\circ}$ & $1 /(4 \cdot 32 J)$ & $0 \cdot 6160$ \\
PFP-1b & Sandwich & $45^{\circ}$ & $1 /(2 J)$ & $0 \cdot 5$ \\
PFP-2b & $45^{\circ}$ & $30^{\circ}$ & $1 /(2 J)$ & $0 \cdot 6123$ \\
\hline
\end{tabular}


quence of figure 1 , for which the spin lock duration is relatively short and the resulting pseudopure state amplitude is comparable with the laboratory frame sequences. ${ }^{3,4}$

Clearly, sequence SL-1b requires one-fourth the mixing time of sequence PFP-1b, to attain the same pseudopure state amplitude $(0.5)$, while SL-2b produces $3.5 \%$ extra amplitude for the same mixing time, with slightly different flip angles. The third sequence SL-3b on the other hand, requires $46 \cdot 3 \%$ of the mixing time required by PFP- $2 b$ and reaches the pseudopure state amplitude $0 \cdot 6160$, representing a marginal $0.6 \%$ amplitude gain.

Further, sequences may also be designed to reach pseudopure state amplitudes that correspond to the unitary bounds on the transformations of states into one another. ${ }^{18-20}$

It may be noted however that homonuclear twospin zero quantum coherence (ZQC) survives the gradient (figure 1) - or any phase cycling. It is to be eliminated for the final state to be a genuine pseudopure state. The final state of the system calculated is shown in the expression given by (4). Equation 4 follows from (3a), recognizing that the $I_{1 x} I_{2 x}$ term which is a mixture of ZQC and double quantum coherence (DQC) of $x$-phase has its DQC component spoiled by the crusher gradient of duration $\tau_{\mathrm{g}}$, while ZQC of $x$-phase processes during this time.

$$
\begin{aligned}
& a\left[\left(I_{1 z}+I_{2 z}\right)-2 I_{1 z} I_{2 z}\right]- \\
& \frac{a}{2}\left[I_{1 y} I_{2 y}+I_{1 x} I_{2 x}\right] \cos \left(\omega_{\Delta} \tau_{g}\right)- \\
& \frac{a}{2}\left[I_{1 y} I_{2 x}-I_{1 x} I_{2 y}\right] \sin \left(\omega_{\Delta} \tau_{g}\right)
\end{aligned}
$$

Here $a$ is the amplitude of the pseudopure state, $\omega_{\Delta}$ being the zero quantum precession frequency in $\mathrm{rad} / \mathrm{s}$. We adopt the following strategy for elimination of homonuclear two-spin zero quantum coherence in the final state.

$2.2 \mathrm{~b}$ Elimination of two-spin homonuclear zero quantum coherence: A spin selective $\pi$ pulse issued before readout converts zero quantum coherence to double quantum coherence: which gets inverted by a $90^{\circ}$ phase shift of this pulse. We may therefore perform two experiments with phase shifted $\pi$ pulses, respectively of phases $x$ and $y$, adding the resulting signals. This removes the $\mathrm{ZQC} / \mathrm{DQC}$ in the final state, producing the desired pseudopure state. Note that the selective inversion pulse also transforms one pseudopure state into another. We have adopted this strategy in our experiments.

Experiment 1:

$$
\begin{aligned}
& I_{1}: \theta_{y}-[S L]_{x}-\phi_{x} \\
& I_{2}: \theta_{-y}-[S L]_{x}-\phi_{-x} 180_{x}^{I_{2}}-90_{y}^{I_{1} / I_{2}}-A c q u . \\
& \text { grad } \\
& a\left[I_{1 z}-I_{2 z}+2 I_{1 z} I_{2 z}\right]-\frac{a}{2}\left[-I_{1 y} I_{2 y}+I_{1 x} I_{2 x}\right] \\
& \cos \left(\omega_{\Delta} \tau_{g}\right)-\frac{a}{2}\left[I_{1 y} I_{2 x}+I_{1 x} I_{2 y}\right] \sin \left(\omega_{\Delta} \tau_{g}\right)
\end{aligned}
$$

Experiment 2:

$$
\begin{aligned}
& I_{1}: \theta_{y}-[S L]_{x}-\phi_{x} \\
& \begin{array}{c}
I_{2}: \theta_{-y}-[S L]_{x}-\phi_{-x} 180_{y}^{I_{2}}-90_{y}^{I_{1} / I_{2}}-A c q u . \\
\operatorname{grad} \\
a\left[I_{1 z}-I_{2 z}+2 I_{1 z} I_{2 z}\right]-\frac{a}{2}\left[I_{1 y} I_{2 y}-I_{1 x} I_{2 x}\right] \\
\cos \left(\omega_{\Delta} \tau_{g}\right)-\frac{a}{2}\left[-I_{1 y} I_{2 x}-I_{1 x} I_{2 y}\right] \sin \left(\omega_{\Delta} \tau_{g}\right)
\end{array}
\end{aligned}
$$

Adding (5) and (6) results in the pseudopure state.

$$
(5)+(6) \Rightarrow 2 a\left[I_{1 z}-I_{2 z}+2 I_{1 z} I_{2 z}\right]
$$

The elimination of ZQC is also possible in a single scan: a suitable crusher gradient after the inversion pulse will result in the desired pseudopure state.

2.2c Quantum state tomography of a two-spin-1/2 system: As an illustration of the quantum state tomography of a pseudopure state in a homonuclear two-spin-1/2 system, we have investigated a pair of equivalent protons in a nematic liquid crystalline environment employing standard methods. The system now corresponds to a three level system, ${ }^{21}$ being a qutrit in effect.

The generation of pseudopure states in a multilevel system that exhibits quadrupolar or dipolar couplings is easily realized experimentally using 
transition selective pulses. ${ }^{22-25}$ For a spin-1 - or two equivalent spins-1/2 - in liquid crystal medium we have three energy levels (corresponding to a single qutrit); two single quantum transitions may be observed in the spectrum, with a splitting of the order of hundreds if not thousands of Hertz, depending on the order parameter.

We may prepare three different pseudopure states in a single qutrit system, ${ }^{26}$ employing the straightforward approach of destroying coherence with a spoiler following a single transition excitation pulse that maximizes coherence, immediately followed if required by an inversion pulse. The pulse sequence is shown in table 4.

$\theta^{j}$. and $\varphi^{i j}$. represent transition selective pulses connecting the two energy levels $|i\rangle$ and $|j\rangle$. To find the flip angle $\theta$ that saturates the transition under consideration one may derive analytical expressions ${ }^{27}$ for the two different single quantum transitions. The transition selective pulse propagators $X_{i j}$ and $Y_{i j}$ of phase $x$ and $y$ respectively are shown in (8) or the two single quantum transitions. For the generation of a pseudopure state (PPS- $|0\rangle$ and PPS- $|2\rangle$ ) from the above propagators we find $\theta=63 \cdot 6^{\circ}$. For the generation of PPS- $|1\rangle$ the pulse $\varphi=127.26^{\circ}$ acts as an inversion pulse, inverting the populations across the corresponding transition.

$$
\begin{aligned}
& X_{01}=\left(\begin{array}{ccc}
\cos \frac{\theta}{\sqrt{2}} & -i \sin \frac{\theta}{\sqrt{2}} & 0 \\
-i \sin \frac{\theta}{\sqrt{2}} & \cos \frac{\theta}{\sqrt{2}} & 0 \\
0 & 0 & 1
\end{array}\right) \\
& X_{12}=\left(\begin{array}{ccc}
1 & 0 & 0 \\
0 & \cos \frac{\theta}{\sqrt{2}} & -i \sin \frac{\theta}{\sqrt{2}} \\
0 & -i \sin \frac{\theta}{\sqrt{2}} & \cos \frac{\theta}{\sqrt{2}}
\end{array}\right) \\
& Y_{01}=\left(\begin{array}{ccc}
\cos \frac{\theta}{\sqrt{2}} & -\sin \frac{\theta}{\sqrt{2}} & 0 \\
\sin \frac{\theta}{\sqrt{2}} & \cos \frac{\theta}{\sqrt{2}} & 0 \\
0 & 0 & 1
\end{array}\right) \\
& Y_{12}=\left(\begin{array}{ccc}
1 & 0 & 0 \\
0 & \cos \frac{\theta}{\sqrt{2}} & -\sin \frac{\theta}{\sqrt{2}} \\
0 & \sin \frac{\theta}{\sqrt{2}} & \cos \frac{\theta}{\sqrt{2}}
\end{array}\right)
\end{aligned}
$$

Table 4. Pulse sequence to generate three different pseudopure states in a single qutrit system.

\begin{tabular}{lc}
\hline Pulse sequence & Pseudopure state \\
\hline$\theta^{12}-$ Grad & PPS- $|0\rangle$ \\
$\theta^{01}-\mathrm{Grad}$ & PPS- -2$\rangle$ \\
$\theta^{12}-\mathrm{Grad}-\varphi^{01}-\mathrm{Grad}$ & PPS- $|1\rangle$ \\
\hline
\end{tabular}

We perform quantum state tomography to characterize the system after generating the pseudopure state. In general, the quantum state of the system is described by the density matrix. The density matrix for a three level system may be represented by a $3 \times 3$ matrix, e.g. (9):

$$
\rho=\left(\begin{array}{ccc}
a & x_{a}+i y_{a} & x_{b}+i y_{b} \\
x_{a}-i y_{a} & b & x_{c}+i y_{c} \\
x_{b}-i y_{b} & x_{c}-i y_{c} & c
\end{array}\right) .
$$

Tomographic reconstruction of the density matrix requires nine elements to be detected. The three diagonal elements $a, b, c$ in (9) could be directly obtained by a single experiment employing a crusher followed by a single hard pulse with small flip angle. We do six additional experiments to find the remaining six elements of the density matrix. ${ }^{28}$ The choice of the unitary operation is such that the elements to be identified in the density matrix are brought into the diagonal position of the matrix followed by a crusher gradient which destroys the unwanted coherences. The diagonal elements are then identified by a small flip angle read pulse. The set of six experiments is performed for each of the three pseudopure states and the thermal equilibrium state.

\subsection{Implementation of $S W A P$ and readout in $1 D$ $\operatorname{mode} e^{15}$}

The logical SWAP operation when performed using isotropic mixing reduces the gate time to $(1 / 2 J)$, and the experimental verification was performed on a two-spin-1/2 system in 2D mode. ${ }^{13}$ Here, we employ 1D and multiplexed 1D approaches and demonstrate the application and performance of selective isotropic mixing on a three-spin-1/2 system.

The functioning of SWAP performed on operational spins may be tracked by monitoring the transitions of the observer spin. On our model AMX spin system we choose the two spins corresponding to the high field multiplets as the operational spins $I_{1}$ 
and $I_{2}$, while the spin corresponding to the low field multiplet arises from the observer spin $I_{0}$ (figure $6 \mathrm{a}$ ). Spin $I_{0}$ exhibits a four line pattern and the four transitions are labeled as line- $a$ to line- $d$ as indicated in figure $6 \mathrm{a}$. We attempt to establish the functioning of SWAP by-performing a short series of 1D experiments. The pulse sequence we have developed is shown in figure 2 ; it is implemented with a two-step phase cycle involving phase alternation of the final $90^{\circ}$ pulse, with no change in the receiver phase.

Note that sequence $b$ effectively involves not only a $270^{\circ}$ pulse rotation of the chosen transition around $y$, but in addition the inversion of spin labels of the coupled spins. This results in 'migration' of the selected multiplet component. The inner lines (lines $b$ and $c$, with coupled spin states $\alpha_{1} \beta_{2}$ and $\beta_{1} \alpha_{2}$ ) are further affected by SWAP in sequence $a$, while the outer lines (lines $a$ and $d$ ) are not (the coupled spin states being $\alpha_{1} \alpha_{2}$ and $\beta_{1} \beta_{2}$ in this case). The detailed dynamics is illustrated by density matrix calculations of the transitions of the observer spin $I_{0}$ for the downfield component and the next higher field component (line- $a$ and line- $b$, respectively) for the sequences with and without SWAP.

Excitation of line- $a$ followed by SWAP (figure 2a.):

$$
\begin{aligned}
& \frac{I_{0 z}}{4}\left[1+2 I_{1 z}+2 I_{2 z}+4 I_{1 z} I_{2 z}\right] \stackrel{90_{y}^{\circ}\left(I_{0}\right)}{\longrightarrow} \\
& \frac{I_{0 x}}{4}\left[\begin{array}{c}
\text { Line- } a \\
1+2 I_{1 z}+2 I_{2 z}+4 I_{1 z} I_{2 z}
\end{array}\right] \stackrel{90_{y}^{\circ}}{\longrightarrow}
\end{aligned}
$$

(a)

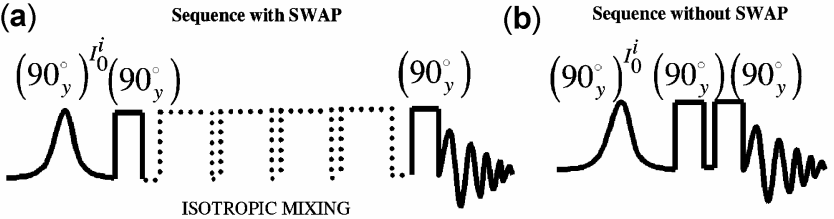

Figure 2. (a) Pulse sequence to implement 1D SWAP. The first soft pulse is a transition selective pulse on observer spin $I_{0}^{i}$, where the superscript ' $i$ ' represents one of the multiplet components of the spin. Transition selectivity is achieved by using a Gaussian pulse of $500 \mathrm{~ms}$ duration. For the hard $90^{\circ}$ pulse we use $8 \mu \mathrm{s}$. The isotropic mixing is performed by a train of $\pi$-pulses each of duration $2 \mathrm{~ms}$ and pulse interval $400 \mu \mathrm{s}$. The mixing time is $1 / 2 J s$, which is $50 \mathrm{~ms}$ in this case. (b) Pulse sequence without SWAP. A two-step phase cycling was implemented, comprising selective pulse phase alternation together with the receiver.

$$
\begin{aligned}
& \frac{-I_{0 z}}{4}\left[1+2 I_{1 x}+2 I_{2 x}+4 I_{1 x} I_{2 x}\right] \stackrel{(S L)_{x},\left(2 J_{12}\right)^{-1}}{\longrightarrow} \\
& \frac{-I_{0 z}}{4}\left[1+2 I_{2 x}+2 I_{1 x}+4 I_{1 x} I_{2 x}\right] \stackrel{90_{y}^{\circ}}{\longrightarrow} \\
& \frac{-I_{0 x}}{4}\left[1-2 I_{2 z}-2 I_{1 z}+4 I_{1 z} I_{2 z}\right] \text { (Line-d) }
\end{aligned}
$$

Excitation of line- $a$, without SWAP (figure $2 b$ ):

$$
\begin{aligned}
& \frac{I_{0 z}}{4}\left[1+2 I_{1 z}+2 I_{2 z}+4 I_{1 z} I_{2 z}\right] \stackrel{90_{y}^{\circ}\left(I_{0}\right)}{\longrightarrow} \\
& \frac{I_{0 x}}{4}\left[\begin{array}{c}
\text { Line- } a \\
1+2 I_{1 z}+2 I_{2 z}+4 I_{1 z} I_{2 z}
\end{array}\right] \stackrel{90_{y}^{\circ}}{\longrightarrow} \\
& \frac{-I_{0 z}}{4}\left[1+2 I_{1 x}+2 I_{2 x}+4 I_{1 x} I_{2 x}\right] \stackrel{90_{y}^{\circ}}{\longrightarrow} \\
& \frac{-I_{0 x}}{4}\left[1-2 I_{1 z}-2 I_{2 z}+4 I_{1 z} I_{2 z}\right](\text { Line- } d)
\end{aligned}
$$

Excitation of line- $b$ followed by SWAP (figure 2a):

$$
\begin{aligned}
& \frac{I_{0 z}}{4}\left[1+2 I_{1 z}-2 I_{2 z}-4 I_{1 z} I_{2 z}\right] \stackrel{90_{y}^{\circ}\left(I_{0}\right)}{\longrightarrow} \\
& \frac{I_{0 x}}{4}\left[\begin{array}{c}
\text { Line- } b \\
1+2 I_{1 z}-2 I_{2 z}-4 I_{1 z} I_{2 z}
\end{array}\right] \stackrel{90_{y}^{\circ}}{\longrightarrow} \\
& \frac{-I_{0 z}}{4}\left[1+2 I_{1 x}-2 I_{2 x}-4 I_{1 x} I_{2 x}\right] \stackrel{(S L)_{x},\left(2 J_{12}\right)^{-1}}{\longrightarrow} \\
& \frac{-I_{0 z}}{4}\left[1+2 I_{2 x}-2 I_{1 x}-4 I_{1 x} I_{2 x}\right] \stackrel{90_{y}^{\circ}}{\longrightarrow} \\
& \left.\frac{-I_{0 x}}{4}\left[1-2 I_{2 z}+2 I_{1 z}-4 I_{1 z} I_{2 z}\right] \text { (Line- } b\right)
\end{aligned}
$$

Excitation of line- $b$, without SWAP (figure $2 \mathrm{~b}$ ):

$$
\begin{aligned}
& \frac{I_{0 z}}{4}\left[1+2 I_{1 z}-2 I_{2 z}-4 I_{1 z} I_{2 z}\right] \stackrel{90_{y}^{\circ}\left(I_{0}\right)}{\longrightarrow} \\
& \frac{I_{0 x}}{4}\left[\begin{array}{c}
\text { Line- } b \\
1+2 I_{1 z}-2 I_{2 z}-4 I_{1 z} I_{2 z}
\end{array}\right] \stackrel{90_{y}^{\circ}}{\longrightarrow}
\end{aligned}
$$




$$
\begin{aligned}
& \frac{-I_{0 z}}{4}\left[1+2 I_{1 x}-2 I_{2 x}-4 I_{1 x} I_{2 x}\right] \stackrel{90_{y}^{\circ}}{\longrightarrow} \\
& \frac{-I_{0 x}}{4}\left[1-2 I_{1 z}+2 I_{2 z}-4 I_{1 z} I_{2 z}\right] \text { (Line-c) }
\end{aligned}
$$

Excitation of line- $a$ leads to line- $d$ and vice versa, regardless of the SWAP operation. However when line- $b$ or line- $c$ is excited, the effect of SWAP is that the intensity returns to the transition excited. The experimental verification is presented in $\$ 3.2$.

\subsection{Implementation of SWAP and readout in multiplexed $1 D$ mode ${ }^{15}$}

We propose another set of experiments with the Hadamard $^{16,29}$ mode of excitation and decoding to improve the sensitivity of the 1D version described above. A multiple selective shaped pulse with suitable multiple modulation provides the desired phase encoding of the multiplet components of the observer spin. We have effected quadruply selective excitation on $I_{0}$, following in turn each of the rows of the Hadamard matrix of order four. The pulse carrier frequency was in each case set to the multiplet center, the two modulation frequencies corresponding to one-fourth the sum and difference of the outer line splitting and the inner line splitting.

The Hadamard matrix of order four is given by:

$$
H=\left[\begin{array}{rrrr}
1 & 1 & 1 & 1 \\
1 & -1 & 1 & -1 \\
1 & 1 & -1 & -1 \\
1 & -1 & -1 & 1
\end{array}\right]
$$

Four experiments were performed with four different modulation schemes, providing the four different modes of phase encoding of the multiplet components. A shaped pulse with suitable cosine and sine modulation is used to generate the desired phase encoding. The encoding scheme we use is shown in table 5 .

The decoding scheme follows the columns in the Hadamard matrix. The experiment time to attain a given sensitivity is hereby reduced to one fourth, in comparison with the time required for the $1 \mathrm{D}$ sequence without the Hadamard strategy.

It may be noted that the performance of any logic operation is most completely described by gate fidelity which may be calculated from a suitable quantum process tomography measurement. ${ }^{30}$
Table 5. The trigonometric identities establishing the encoding pattern which follows the rows in the Hadamard matrix.

\begin{tabular}{lc}
\hline Modulation scheme & Encoding of the peaks \\
\hline Cosine-Cosine & ++++ \\
Sine-Cosine & +-+- \\
Cosine-Sine & ++-- \\
Sine-Sine & +--+ \\
\hline
\end{tabular}

\section{Results and discussion}

\subsection{Preparation of pseudopure states}

3.1a Heteronuclear two-spin-1/2 system: We have tested our sequences on a heteronuclear two AX spin system. A sample of formic acid $\left(J_{\mathrm{AX}}=219.16 \mathrm{~Hz}\right)$ with $99 \%{ }^{13} \mathrm{C}$, in $\mathrm{C}_{6} \mathrm{D}_{6}$ solvent was used. The generation of the pseudopure state is verified by performing readout on both ${ }^{1} \mathrm{H}$ and ${ }^{13} \mathrm{C}$ channels. The experimental spectra are shown in figure 3 .

The ratio of amplitudes in the thermal equilibrium spectrum is $1: 1$ for both the nuclei. The theoretical amplitude of the pseudopure state spectrum is $2 \cdot 5: 0$ and $0.625: 0$ for ${ }^{13} \mathrm{C}$ and ${ }^{1} \mathrm{H}$ spins respectively. The respective experimental peak amplitude ratios obtained by our method is $2.23:-0.15$ and $0.57:-0.03$. The laboratory frame counterpart gives 2.35:0.29 and $0.59: 0 \cdot 12$ respectively. Note in both cases the significantly larger 'contamination' in the pseudopure states obtained by the laboratory frame sequences.

Grover's algorithm was implemented on the same heteronuclear sample as above. The pseudopure state was created by our rotating frame sequence (SL-1a). The quality of the results is comparable to the corresponding laboratory frame approach with temporal averaging. ${ }^{31}$

3.1b Homonuclear two spin-1/2 system: We have also experimentally tested the generation of pseudopure states on homonuclear two spin-1/2 systems. The sample used is cinnamic acid in DMSO- $d_{6}$. The aliphatic trans-protons being used as qubits have a coupling constant of $16 \mathrm{~Hz}$. As pointed out earlier, the thermal equilibrium state of the system is symmetric and segment $\mathbf{B}$ of the pulse sequence in figure 1 is used as the starting point to create the pseudopure state in this case. The experiment is performed for $\theta=\phi=45^{\circ}, t=1 / 8 J$ [SL-1b]. The readout is performed on both the spins. The $\theta$ and $\varphi$ pulses of different phases are realized as follows, the 
(a)

$\begin{array}{llllllllllll}2.5 & 2.0 & 1.5 & 1.0 & 0.5 & 0.0 & -0.5 & -1.0 & -1.5 & -2.0 & -2.5 & \mathrm{ppm}\end{array}$ (b)

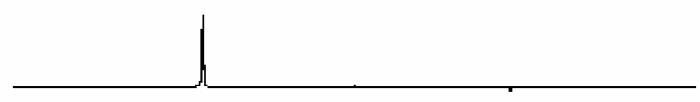

Figure 3. Spectra resulting from pseudopure state of heteronuclear AX spin system generated employing the pulse sequence SL-1a of figure 1. (Arbitrary 'shift' scale.) The experiment was performed for $\theta=\phi=45^{\circ}, t=1 / 4 J_{A X}$. Readout is performed on ${ }^{13} \mathrm{C}$ and ${ }^{1} \mathrm{H}$ channels. (a) and (b) are ${ }^{13} \mathrm{C}$ and ${ }^{1} \mathrm{H}$ spectra. Other sequence parameters as in the caption to figure 1.

(a)

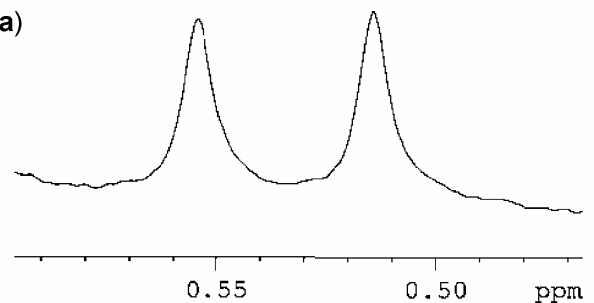

(c)

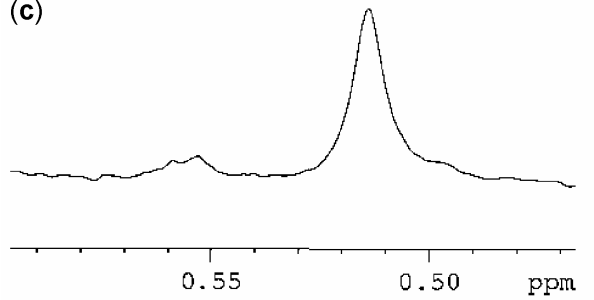

(b)

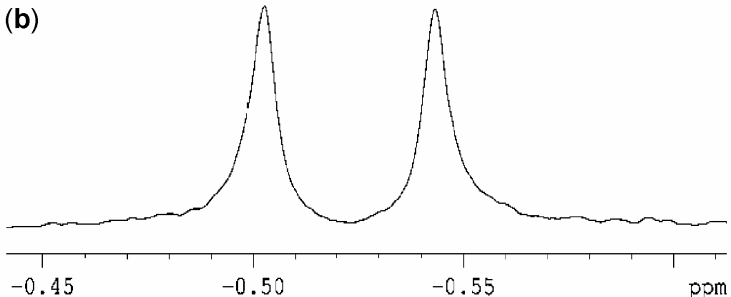

(d)

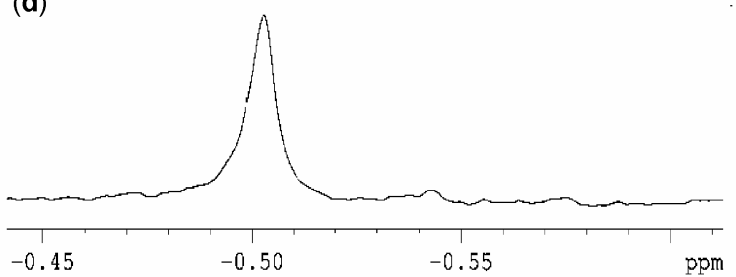

Figure 4. Spectra from homonuclear AX spin system. (a) and (b) are thermal equilibrium spectra of low field and high field doublet components respectively. (c) and (d) are corresponding low and high field spectra obtained after the generation of pseudopure state (sequence SL-1b). (Arbitrary 'shift' scale.) The experiment was performed for $\theta=\phi=45^{\circ}, t=1 / 8 \mathrm{~J}$. $\left(4 \Delta \delta^{-1}=589.43 \mu \mathrm{s}\right.$ in the present case. We use a $17 \mu \mathrm{s}, 90^{\circ}$ hard pulse on ${ }^{1} \mathrm{H}$ channel. Isotropic mixing is performed using DIPSI-2 sequence. The readout is performed with a $6 \mathrm{~ms}$ spin selective Gaussian pulse. Other sequence parameters as in the caption to figure 1 .

transmitter offset being at the mean chemical shift of the two spins:

$$
\begin{aligned}
& I_{1}: \theta_{y} \\
& I_{2}: \theta_{-y}: 90_{x}-\frac{1}{4(\Delta \delta)}-90_{-x}, \\
& I_{1}: \varphi_{x} \\
& I_{2}: \varphi_{-x}: 90_{y}-\frac{1}{4(\Delta \delta)}-90_{-y},
\end{aligned}
$$

$I_{1}$ and $I_{2}$ represent the two spins with chemical shift difference $\Delta \delta$. The spectra are presented in figure 4 .

The ratio of amplitudes of the thermal equilibrium spectrum is $1: 1$ for both the spins. The experimental amplitude ratio by our method for the high field doublet is $0.93: 0.04$ and for the low field doublet is $0.25: 1.04$. Note that different components are picked in the two doublets owing to the effect of the spin selective $\pi$ pulse employed for suppression of zero quantum coherence. The laboratory frame sequence gives $-0.7979: 0.26$ for high field doublet and $-0.6690:-0.2975$ for the low field doublet 
(a)

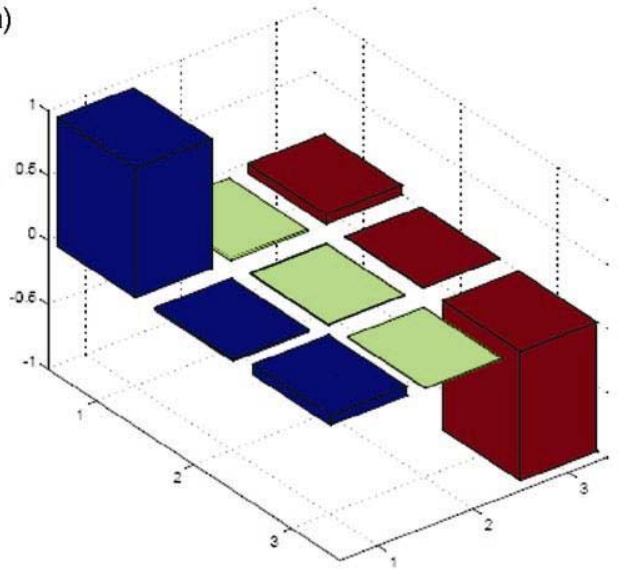

(c)
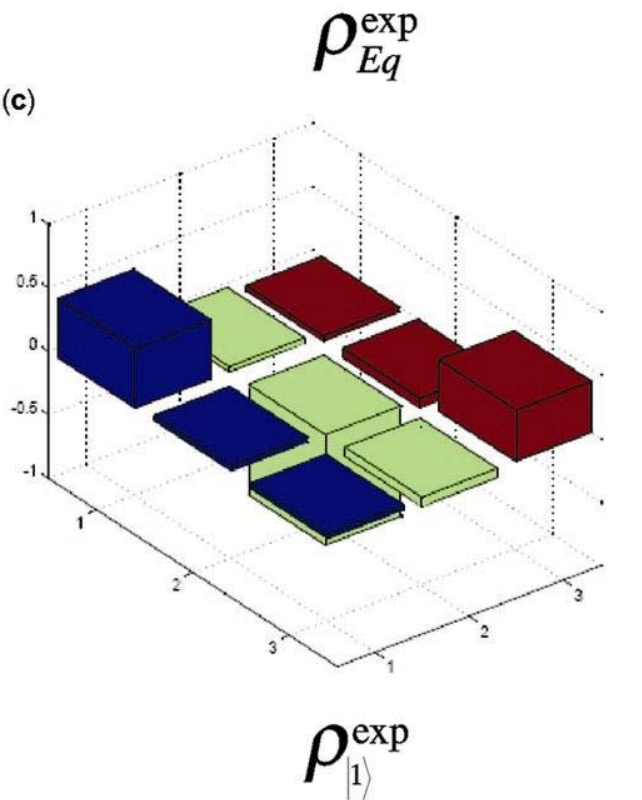

(b)

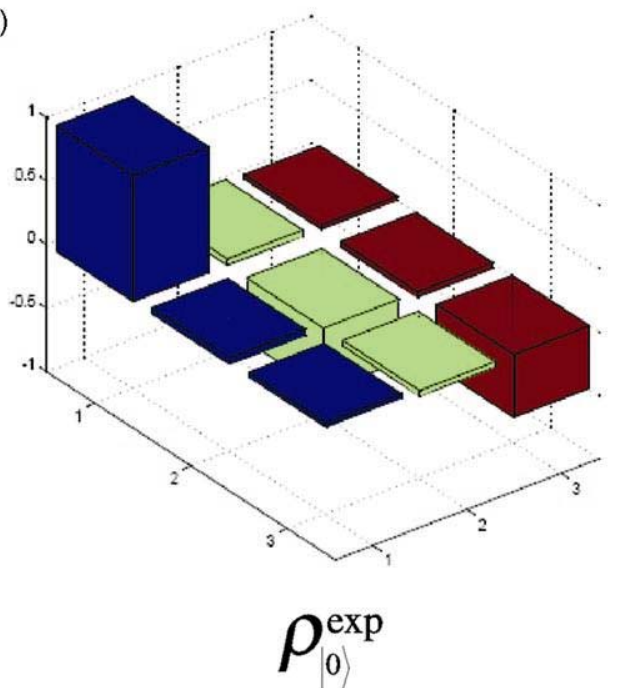

(d)

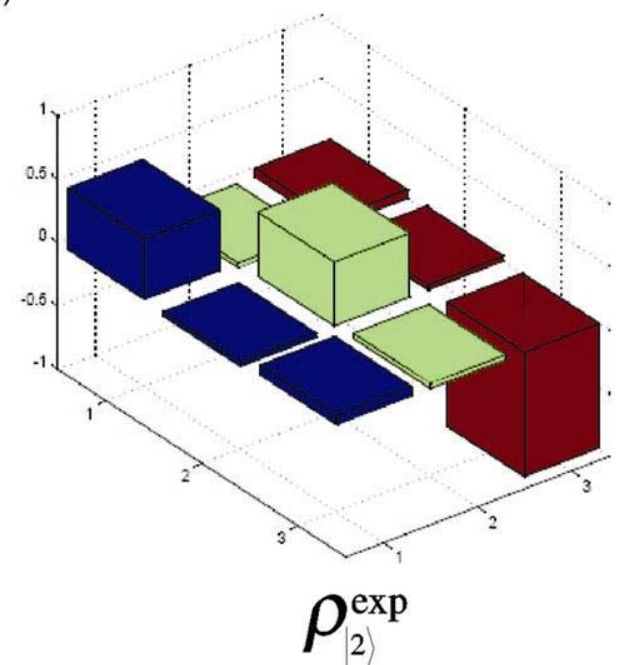

Figure 5. Quantum state tomography on $\mathrm{CH}_{2} \mathrm{Cl}_{2}$ in EBBA. (a) Thermal equilibrium. Three different pseudopure states generated are shown in (b), (c) and (d). The plot describes only the real part of the density matrix.

respectively. The time duration for the generation of pseudopure state is $7.81 \mathrm{~ms}$ in this case. On the other hand, both laboratory frame sequences require a time duration of $31.25 \mathrm{~ms}$. The significant improvement in the amplitude and 'purity' of the pseudopure state obtained with the rotating frame sequence may be underscored.

3.1c Quantum state tomography on a two-spin-1/2 system: We have employed $\mathrm{CH}_{2} \mathrm{Cl}_{2}$ in EBBA as the sample. Probing the system in thermal equilibrium, we get two lines of equal intensity separated by $4076 \cdot 34 \mathrm{~Hz}$ at $30^{\circ} \mathrm{C}$. Being a three level system, ${ }^{21}$ three different pseudopure states could be prepared by equalizing the populations in any two of the levels. The pulse sequence used has been shown in table 4 . We then perform quantum state tomography to map all the elements in the density matrix. The peak intensities are the measure of the population differences; employing the additional condition that the trace of the density matrix is zero results in three linear equations from which we get the diagonal elements of the density matrix. As described earlier we perform six additional experiments and by solving the resulting equations we determine the off-diagonal elements in the density matrix. 
(a)

(b)

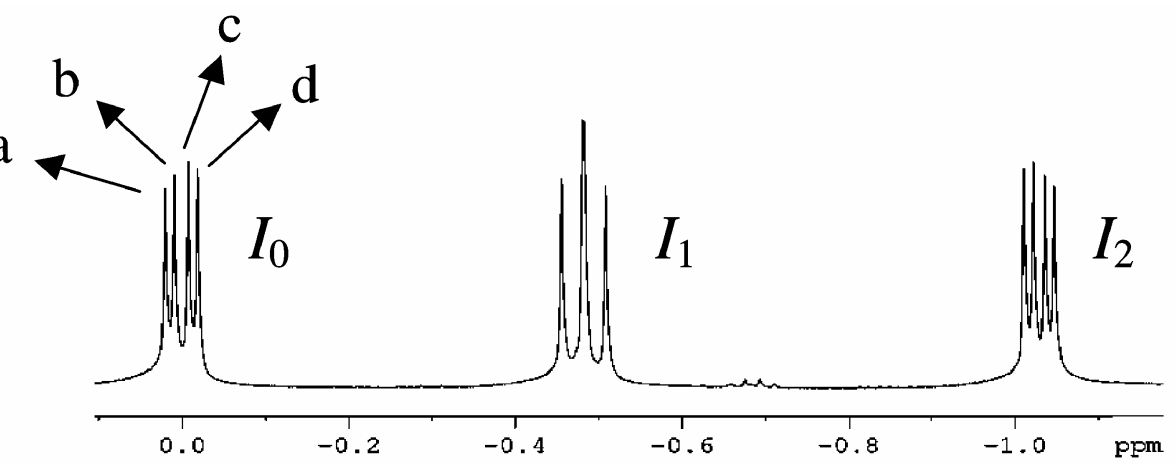

Line-a excited

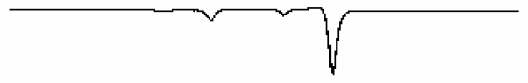

Line-b excited

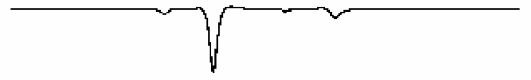

Line-c excited

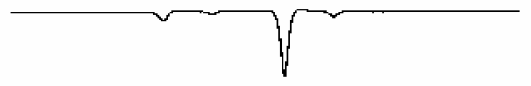

Line-d excited

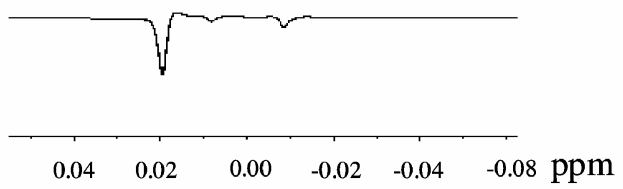

(c)

Line-a excited

Line-b excited

Line-c excited
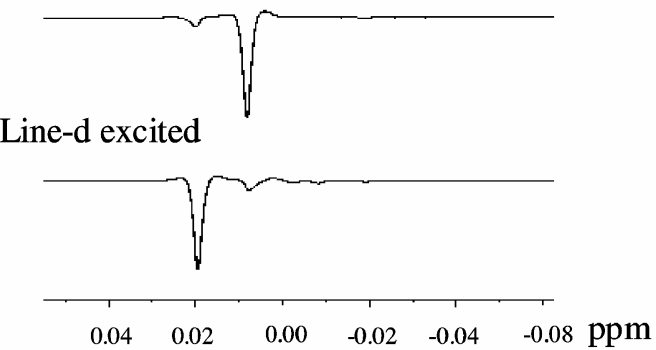

Figure 6. (a) 1D spectrum of 2,3-dibromopropionic acid. The labelling of the multiplet components of the observer spin $\left(I_{0}\right)$ is indicated. (b) 1D Spectra with SWAP. (c) 1D spectra without SWAP. (Arbitrary 'shift' scale.) Transition selective excitation is achieved employing a Gaussian pulse of $500 \mathrm{~ms}$ duration. Other sequence parameters as in figure 2.

Thus we can identify the state of the system completely by performing seven experiments in all. The plot of the real part of the density matrix elements is shown in figure 5 . The imaginary part is identically zero.

\subsection{Implementation of SWAP and readout in $1 D$ mode}

The SWAP readout performed in 1D fashion has the advantage of significant reduction in the readout time. The experiment was performed on the sample 2,3-dibromopropionic acid (AMX, here denoted $I_{1} I_{2} I_{0}$ ). The coupling constants are $J_{01}=11.05 \mathrm{~Hz}, J_{02}=$ $4.53 \mathrm{~Hz}, J_{12}=10.02 \mathrm{~Hz}$. Spin $\mathrm{X}$ (which gives rise to the lowest field multiplet) is used as the observer spin $I_{0}$ while the spins $\mathrm{A}$ and $\mathrm{M}$ are used as operational spins $\left(I_{1}, I_{2}\right)$.
The spectra obtained for the sequence with and without SWAP are shown in figures $6 \mathrm{~b}$ and $\mathrm{c}$ respectively, the normal $1 \mathrm{D}{ }^{1} \mathrm{H}$ NMR spectrum being displayed in figure $6 \mathrm{a}$ for reference.

Four experiments were performed, exciting one multiplet component of the observer spin in each. Excitation of line- $a$ leads to line- $d$ and vice versa, regardless of the SWAP operation. However when line- $b$ or line- $c$ is excited, the effect of SWAP is seen as expected: intensity migrates to the other component without SWAP, while with SWAP intensity returns to the transition excited.

When performed in a conventional 2D mode the experiment time is typically two orders of magnitude higher. Note that in the spectra with SWAP (figure $6 \mathrm{~b}$ ) we lose some signal intensity, owing to relaxation losses during the extended isotropic mixing period. 

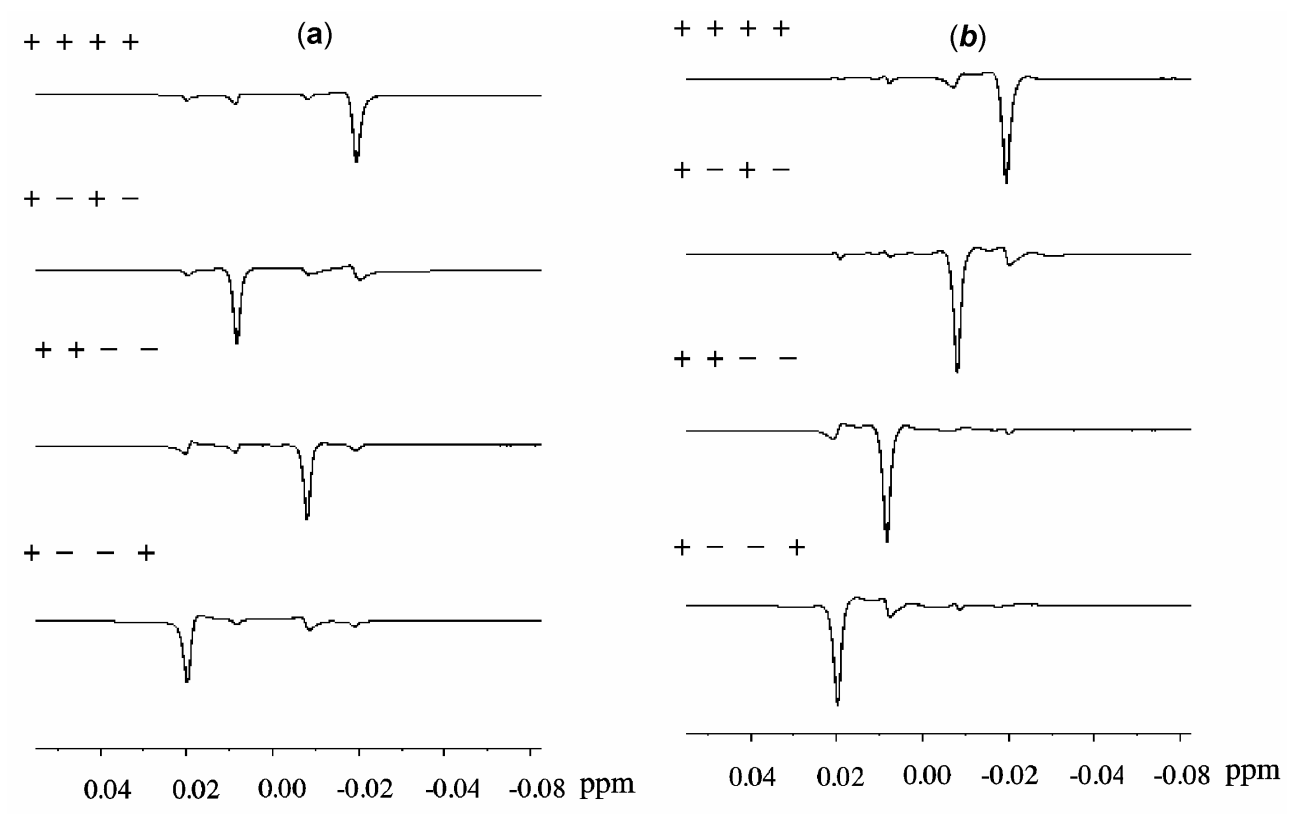

Figure 7. Spectra of 2,3-dibromopropionic acid. (a) Decoded spectra with SWAP. (b) Decoded spectra without SWAP. (Arbitrary 'shift' scale.) Here the transition selective excitation is achieved employing a modulated Gaussian pulse of $500 \mathrm{~ms}$ duration. The modulation scheme follows the rows in the Hadamard matrix. The decoding scheme follows the column in the Hadamard matrix. Other sequence parameters as in figure 2 .

\subsection{Implementation of $S W A P$ and readout in multiplexed $1 D$ mode}

The 1D SWAP described in $\$ 2.3$ can be performed in a $1 \mathrm{D}$ multiplexed mode. In this present case which involves four transitions in each multiplet, the experiment is performed in one-fourth of the time required by the single transition selective mode of the experiment, to attain a given sensitivity. The decoded spectra are shown for the experiments with and without SWAP operation in figures $7 \mathrm{a}$ and $\mathrm{b}$ respectively.

Here we perform four experiments with the multiplet components of the observer spin $\left(I_{0}\right)$ being phase encoded in accordance with the rows of the Hadamard matrix (table 5) of order four. The decoding scheme follows the columns of the Hadamard matrix, and is shown at the top of each spectrum. Note that the spectra exhibit the same patterns as in figures $6 \mathrm{~b}$ and $\mathrm{c}$, respectively.

The measured sensitivities with and without the multiplexed mode in the 1D SWAP operation are comparable for the corresponding experiments. It may be noted however that the experimental time for the multiplexed mode is reduced by a factor of four.

\section{Conclusion}

We have reported some general approaches in ensemble quantum computing. Specifically, we have demonstrated the advantages to be gained by the application of rotating frame coherence transfer sequences for 'initialization' of the NMR spin ensembles, as well as for gating as exemplified by the SWAP operation. We have also demonstrated a 1D and multiplexed 1D approach to the readout process for the SWAP operation. The extension of our work to larger networks of coupled spins and to multilevel systems is in progress.

\section{Acknowledgement}

The authors acknowledge useful discussions with Ms N Ananth during the early stages of this work. We gratefully acknowledge Council of Scientific and Industrial Research (CSIR) for an extramural research grant to N C and grant of a Junior Research 
Fellowship to V V and the Department of Science and Technology (DST) for grant of an AV 400 spectrometer to the Department.

\section{References}

1. Ernst R R, Bodenhausen G and Wokaun A 1987 Principles of nuclear magnetic resonance in one-and two-dimensions (Oxford: Clarendon Press)

2. Cory D G, Fahmy A F and Havel T F 1997 Proc. Natl. Acad. Sci. USA 941634

3. Pravia M, Fortunato E, Weinstein Y, Price M D, Teklemariam G, Nelson R J, Sharf Y, Samaroo S, Tseng C H, Havel T F and Cory D G 1999 Concepts in magnetic resonance $\mathbf{1 1} 225$

4. Cory D G, Price M D and Havel T F 1998 Physica D120 82

5. Chuang IL, Vandersypen L M K, Zhou X, Leung D W and Lloyd S 1998 Nature (London) 393 143

6. Das R, Mahesh T S and Kumar A 2003 Phys. Rev. A67 062304

7. Grover L K 1997 Phys. Rev. Lett. 794709

8. Chuang L, Gershenfeld N and Kubinec N 1998 Phys. Rev. Lett. 803408

9. Mádi Z L, Brüschweiler R and Ernst R R $1998 \mathrm{~J}$. Chem. Phys. 10910603

10. Linden N, Barjat H, Carbajo R J and Freeman R 1999 Chem. Phys. Lett. 307198

11. Chandrakumar N and Subramanian S 1986 Modern techniques in high resolution FT NMR (New York: Springer)

12. Dirac P A M 1935 Principles of quantum mechanics (Oxford: Clarendon)

13. Schulte-Herbrüggen T and Sørensen O W 2000 Concepts in magnetic resonance $\mathbf{1 2} 389$

14. Frydman L, Lupulescu A and Scherf T $2003 \mathrm{~J}$. Am. Chem. Soc. 1259204

15. Ananth N, Vimalan V and Chandrakumar N 2005 Rotating frame experiments for $1 D$ and $2 D$ NMR quan- tum computing XXI International Conference on Magnetic Resonance in Biological System, Hyderabad

16. Kupče E, Nishida T and Freeman R 2003 Progress in nuclear magnetic resonance spectroscopy 4295

17. Chandrakumar N and Kimmich R 1999 J. Magn. Reson. 137100

18. Untidt T S and Nielsen N C 2000 J. Chem. Phys. 113 8464

19. Schulte-Herbrüggen $T$, Spörl $A$, Khaneja $N$ and Glaser S J 2005 Phys. Rev. A72 042331

20. Khaneja N, Reiss T, Kehlet C, Schulte-Herbrüggen T and Glaser S 2005 J. Magn. Reson. 172296

21. Samulski E and Tobolsky A V 1969 Mol. Crys. 7 433

22. Khitrin K and Fung B M 2000 J. Chem. Phys. 112 6963

23. Visalakshi V and Chandrakumar N 2001 Quantum information processing with spin systems involving spin-1 nuclei 7th National Magnetic Resonance Society Symposia, Chennai

24. Khitrin K and Fung B M 2001 Phys. Rev. A64 032306

25. Khitrin K, Sun H and Fung B M 2001 Phys. Rev. A63 020301

26. Das R, Mitra A, Kumar V and Kumar A 2003 Int. J. Quant. Inf. 1387

27. Chandrakumar N 1996 Spin-1 NMR NMR: Basic principles and progress (eds) H Günther and E Fluck (Berlin: Springer-Verlag), vol. 34

28. Bonk F A, Sarthour R S, deAzevedo E R, Bulnes J D, Mantovani G L, Freitas J C C, Bonagamba T J, Guimaraes A P and Oliveira S 2004 Phys. Rev. A69 042322

29. Gopinath T and Kumar A 2006 J. Magn. Reson. 183 259

30. Childs A M Chuang I L and Leung D B 2001 Phys. Rev. A64 012314

31. Yannoni C S, Sherwood M H, Miller D C, Chuang I L, Vandersypen L M K and Kubinec M G 1999 Appl. Phys. Lett. 753563 